\title{
PSEUDOINVERSION OF DEGENERATE METRICS
}

\author{
C. ATINDOGBE, J.-P. EZIN, and JOËL TOSSA
}

\author{
Received 21 January 2003
}

\begin{abstract}
Let $(M, g)$ be a smooth manifold $M$ endowed with a metric $g$. A large class of differential operators in differential geometry is intrinsically defined by means of the dual metric $g^{*}$ on the dual bundle $T M^{*}$ of 1 -forms on $M$. If the metric $g$ is (semi)-Riemannian, the metric $g^{*}$ is just the inverse of $g$. This paper studies the definition of the above-mentioned geometric differential operators in the case of manifolds endowed with degenerate metrics for which $g^{*}$ is not defined. We apply the theoretical results to Laplacian-type operator on a lightlike hypersurface to deduce a Takahashi-like theorem (Takahashi (1966)) for lightlike hypersurfaces in Lorentzian space $\mathbb{R}_{1}^{n+2}$.
\end{abstract}

2000 Mathematics Subject Classification: 53C50, 53B30.

1. Introduction. The introduction of a Riemannian metric on a smooth manifold gives rise to a series of differential operators which reveal deep relationships between the geometry and the topology of the manifold. If $\Delta^{k}$ is the Laplacian on $k$-forms, it is well known that, on a compact manifold, its spectrum $\left\{\lambda_{i}^{k}\right\}$ contains topological and geometric information on the manifold. According to the Hodge decomposition theorem, the dimension of the kernel of $\Delta^{k}$ equals the $k$ th Betti number so that the Laplacian determines Euler characteristic $\chi(M)$ of compact Riemannian manifolds $(M, g)$.

Among usual differential operators on $(M, g)$, the exterior derivative which takes $k$-forms to $(k+1)$-forms is the only one defined in terms of the smooth structure of the manifold $M$. The others are defined by means of the metric $g^{*}$ on the dual bundle, which is the inverse of the given metric $g$, in semiRiemannian case.

Many situations arise in mathematical physics where the metric is degenerated and it is not possible to define the inverse $g^{*}$. A typical case is the one we have in the coupling of Einstein theory of gravity with both a quantum mechanics particle with spin and an electromagnetic field, that is, Einstein-DiracMaxwell (EDM) system. If, for instance, we consider Lorentz framed manifold $\bar{M}$ endowed with Eddington-Finkelstein metric (cf. Hawking and Ellis [12, page 150])

$$
d s^{2}=-\left(1-\frac{2 m}{r}\right) d u^{2}+2 d u d r+r^{2}\left(d \theta^{2}+\sin ^{2} \theta d \phi^{2}\right)
$$


given in a coordinate system $(u, r, \theta, \phi)$, where $u=t+\bar{r}$ is an advanced null coordinate with

$$
\bar{r}=\int \frac{d r}{1-2 m / r}=r+2 m \ln (r-2 m) \quad(r>2 m),
$$

the hypersurface $M: u=$ constant is a degenerate hypersurface. Dirac operator can be written outside $M$ and in its interior region. But it is not easy to match the two operators on $M$ due to the fact that the inverse metric $g^{*}$ cannot be defined on $M$. Normalization problems and the geometry of those manifolds are considered in several papers (see $[1,2,3,5,6,7,8,10,11,13,14]$ and references therein).

In the following, we consider a smooth (semi)-Riemannian manifold $(M, g)$, its Levi-Civita connection $\nabla$, its tangent (resp., cotangent) bundle $T M$ (resp., $\left.T M^{*}\right)$, and $\mathscr{F}(M)$ the space of smooth functions on $M$. For a vector bundle $E$, we denote by $\Gamma(E)$ the space of its smooth sections. For a smooth function $f$ in $\mathscr{F}(M)$, the gradient of $f$ is the vector field $\operatorname{grad}^{g} f$ given by

$$
g\left(\operatorname{grad}^{g} f, X\right)=X(f)=d f(X)
$$

for any $X$ in $\Gamma(T M)$.

In local coordinates $\left(x^{1}, \ldots, x^{n}\right)$, the gradient of $f$ is given by

$$
\operatorname{grad}^{g} f=g^{i j} f_{i} E_{j}
$$

where $f_{i}=\partial f / \partial x^{i}, E_{j}=\partial / \partial j$, and $g^{i j}$ is the $(i, j)$-entry of the inverse $g^{-1}$ of g.

The gradient can be defined using exterior differential $d$ and the natural isomorphism \# between the tangent bundle $T M$ and its dual bundle $T M^{*}$, by the composition

$$
\operatorname{grad}: \mathscr{F}(M) \stackrel{d}{\longrightarrow} T M^{*} \stackrel{\#}{\longrightarrow} T M,
$$

that is,

$$
\operatorname{grad}=\# \circ d,
$$

where

$$
\begin{aligned}
\#: T M^{*} & \longrightarrow T M, \\
\omega & \longmapsto \omega^{\#}
\end{aligned}
$$

is such that, for all $Y \in \Gamma(T M)$,

$$
g\left(\omega^{\#}, Y\right)=\omega(Y) .
$$


The inverse of \# is the isomorphism $b=(\#)^{-1}$ defined by

$$
\begin{aligned}
b: T M & \longrightarrow T M^{*}, \\
X & \longmapsto X^{b}
\end{aligned}
$$

such that

$$
X^{b}(Y)=g(X, Y)
$$

for all $Y$ in $\Gamma(T M)$.

The divergence of a vector field $X$ is the codifferential of the dual 1-form $X^{b}$ defined by

$$
\operatorname{div} X=\delta\left(X^{b}\right)=-\operatorname{trace}\left(Z \longmapsto \nabla_{Z} X\right)
$$

The covariant differentiation $\nabla$ defined on vector fields can be extended to 1-forms by duality using isomorphism \#:

$$
\nabla \alpha(X, Y)=g\left(\nabla_{X} \alpha^{\#}, Y\right) \quad \forall \alpha \in T^{*} M
$$

The Laplace-Beltrami operator $\Delta^{g}$, whose physical and mathematical importance is well known, is defined on smooth functions by

$$
\Delta^{g}=\operatorname{div}(\operatorname{grad})=\delta \circ b \circ \# \circ d,
$$

that is, locally,

$$
\Delta_{g}=\sum_{i, j} g^{i j}\left\{\frac{\partial^{2} f}{\partial x^{i} \partial x^{j}}-\Gamma_{i j}^{k} \frac{\partial f}{\partial x^{k}}\right\}
$$

From (1.4) and (1.14), it is obvious that the entries $g^{i j}$ of the inverse of $g_{i j}$ need to be computed in local coordinates. So the following question seems to be coming out in a natural way: how can we define those differential operators on a lightlike manifold, that is, on a submanifold with a degenerate (noninvertible) metric $g$ ?

This question will be answered by Proposition 3.1.

First, note that $\left(d x^{i}\right)$ and $\left(\partial_{i}^{b}\right)$ are two local bases of $T^{*} M$ and the decomposition

$$
\partial_{i}^{b}=\lambda_{i j} d x^{j}, \quad 1 \leq i \leq n .
$$

holds.

Hence,

$$
g_{i k}=g\left(\partial_{i}, \partial_{k}\right)=\partial_{i}^{b}\left(\partial_{k}\right)=\lambda_{i j} d x^{j}\left(\partial_{k}\right)=\lambda_{i k},
$$


and then,

$$
\partial_{i}^{b}=g_{i k} d x^{k}, \quad 1 \leq i \leq n \text {. }
$$

So inverting $g$, we get

$$
d x^{k}=g^{i k} \partial_{i}^{b}, \quad 1 \leq k \leq n
$$

Thus, coefficients $g^{i j}$ appear as the entries of the matrix of transition from basis $\left(\partial_{i}^{b}\right)$ to $\left(d x^{k}\right)$.

Then, an important step in answering the above question should be the construction of the isomorphisms \# and $b$ adapted to the case of lightlike manifolds.

Below, we discuss lightlike submanifolds of semi-Riemannian manifolds. We are particularly interested in lightlike hypersurfaces. One may proceed similarly in any other codimension. The paper is organized as follows. Section 2 deals with preliminaries and specifies our notations and some basic results. Section 3 is devoted to the definition and construction of the pseudoinverse of a degenerate metric $g$, and a concept of associate metric with the degenerate metric $g$ is also introduced. In Section 4, the compatibility of this associate metric with respect to the connection induced by $g$ is examined. The existence and the properties of the pseudoinverse allow the definition of a Laplacian $\Delta^{g}$ on smooth functions of $(M, g)$. Actually, this $\Delta^{g}$ is a d'Alembertian since $g$ is nondefinite. However, for the sake of simplicity, we call it Laplacian and we denote it by $\Delta^{g}$. It is formally defined in the same way as the Laplace operator in Section 5. The Laplacian of lightlike hypersurfaces endowed with parallel screen distribution is then defined and its action on position vector field is expressed (see Theorem 5.2). Finally, in Section 6, we give an application which oddly reminds us of Takahashi theorem [15]. It is our Theorem 6.1.

2. Preliminaries on lightlike hypersurfaces. Let $M$ be a hypersurface of an $(n+2)$-dimensional semi-Riemannian manifold $(\bar{M}, \bar{g})$. In the classical theory of nondegenerate hypersurface, the normal bundle has trivial intersection $\{0\}$ with the tangent one and plays an important role in the introduction of main geometric objects. In case of degenerate hypersurface, the situation is totally different. The normal bundle $T M^{\perp}$ is a rank-one distribution over $M: T M^{\perp} \subset$ $T M$; hence, the metric $g$ on $M$ is degenerate, that is, noninvertible and of rank $n$. In fact, the following propositions is proved in [11]

Proposition 2.1. Let $(M, g)$ be a hypersurface of an $(n+2)$-dimensional semi-Riemannian manifold $(\bar{M}, \bar{g})$. Then the following assertions are equivalent:

(i) $M$ is a lightlike hypersurface of $\bar{M}$;

(ii) $g$ has a constant rank $n$ on $M$;

(iii) $T M^{\perp}=\cup_{x \in M} T_{x} M^{\perp}$ is a distribution on $M$. 
A complementary bundle of $T M^{\perp}$ in $T M$ is a rank- $n$ nondegenerate distribution over $M$. It is called a screen distribution in the tangent bundle and is often denoted by $S(T M)$. In [11, Chapter 4], degenerate hypersurface $(M, g)$ endowed with this specific distribution is denoted by $(M, g, S(T M))$ and is also called lightlike hypersurface. For the convenience of the reader, we summarize the properties of lightlike hypersurfaces which are appropriate to our purpose.

There exists a vector bundle $\operatorname{tr}(T M)$ of rank one over $M$ such that for any nonzero section $\xi$ of $T M^{\perp}$ on a coordinate neighbourhood $U \subset M$, there is a unique section $N$ of $\operatorname{tr}(T M)$ defined on $U$ such that

$$
\overline{\mathfrak{g}}(\xi, N)=1, \quad \overline{\mathfrak{g}}(N, N)=\overline{\mathfrak{g}}(N, W)=0 \quad \forall W \in \Gamma\left(\left.S(T M)\right|_{\varkappa}\right) .
$$

Let $\bar{\nabla}$ and $\nabla$ be the Levi-Civita connection of $(\bar{M}, \bar{g})$ and an induced connection on $(M, g, S(T M))$, respectively. It is known that $\nabla$ is not unique. It depends on both $g$ and the considered screen distribution.

With the decompositions into orthogonal direct sums

$$
\begin{gathered}
T M=S(T M) \perp T M^{\perp}, \\
\left.T \bar{M}\right|_{M}=S(T M) \perp\left(T M^{\perp} \oplus \operatorname{tr}(T M)\right)=T M \oplus \operatorname{tr}(T M),
\end{gathered}
$$

we have Gauss and Weingarten formulae in the form

$$
\begin{aligned}
& \bar{\nabla}_{X} Y=\nabla_{X} Y+h(X, Y) \quad \forall X, Y \in \Gamma(T M), \\
& \bar{\nabla}_{V} X=-A_{V} X+\nabla_{X}^{t} V \quad \forall X \in \Gamma(T M), \forall V \in \Gamma(\operatorname{tr}(T M)),
\end{aligned}
$$

or equivalently,

$$
\begin{aligned}
& \bar{\nabla}_{X} Y=\nabla_{X} Y+B(X, Y) N, \\
& \bar{\nabla}_{X} N=-A_{N} X+\tau(X) N,
\end{aligned}
$$

where we put

$$
\begin{aligned}
B(X, Y) & =\bar{g}(h(X, Y), \xi) \quad \forall X, Y \in \Gamma\left(\left.T M\right|_{\iota}\right), \\
\tau(X) & =\bar{g}\left(\nabla_{X}^{t} N, \xi\right) \quad \forall X \in \Gamma\left(\left.T M\right|_{\varkappa}\right) .
\end{aligned}
$$

For $\nabla_{X} Y$ and $A_{V} X$ belonging to $\Gamma(T M)$ and $h$ a $\Gamma(\operatorname{tr}(T M))$-valued symmetric $\mathscr{F}(M)$-bilinear form on $\Gamma(T M), A_{V}$ is an $\mathscr{F}(M)$-linear operator on $\Gamma(T M)$ and $\nabla^{t}$ is a linear connection on the lightlike transverse vector bundle $\operatorname{tr}(T M)$.

Let $P$ denote the projection morphism of $\Gamma(T M)$ on $\Gamma(S(T M))$ with respect to the decomposition (2.2). We have

$$
\begin{gathered}
\nabla_{X} P Y=\nabla_{X}^{*} P Y+h^{*}(X, P Y) \quad \forall X, Y \in \Gamma(T M), \\
\nabla_{X} U=-A_{U}^{*} X+\nabla_{X}^{* t} U \quad \forall X \in \Gamma(T M), \forall U \in \Gamma\left(T M^{\perp}\right),
\end{gathered}
$$


where $\nabla_{X}^{*} P Y$ and $A_{U}^{*} X$ belong to $\Gamma(S(T M)), \nabla^{*}$ and $\nabla^{* t}$ are linear connections on $S(T M)$ and $T M^{\perp}$, respectively, $h^{*}$ is a $\Gamma\left(T M^{\perp}\right)$-valued $\mathscr{F}(M)$-bilinear form on $\Gamma(T M) \times \Gamma(S(T M))$, and $A_{U}^{*}$ is a $\Gamma(S(T M))$-valued $\mathscr{F}(\mathcal{U})$-linear operator on $\Gamma(T M)$. They are the second fundamental form and the shape operator of the screen distribution $S(T M)$, respectively. Note that some authors called $A_{U}^{*}$ (or $\left.A_{N}\right)$ the Burali-Forti affinor [3, 9].

Define the following also on $U$ :

$$
\begin{aligned}
C(X, P Y) & =\bar{g}\left(h^{*}(X, P Y), N\right), \\
\varepsilon(X) & =\bar{g}\left(\nabla_{X}^{* t} \xi, N\right) .
\end{aligned}
$$

One can verify that $\varepsilon(X)=-\tau(X)$. Thus, locally, one has

$$
\begin{aligned}
\nabla_{X} P Y & =\stackrel{*}{\nabla}_{X} P Y+C(X, P Y) \xi \\
\nabla_{X} \xi & =-A_{\xi}^{*} X-\tau(X) \xi, \quad \forall X \in \Gamma(T M) .
\end{aligned}
$$

DEFINITION 2.2. The screen distribution $S(T M)$ is said to be parallel with respect to the induced connection $\nabla$ if $\nabla_{X} P Y \in \Gamma(S(T M))$ for all $X, Y \in \Gamma(T M)$.

We will make use of the following theorem.

THeOrem 2.3 (Duggal and Bejancu [11, page 89]). Let $(M, g, S(T M))$ be a lightlike hypersurface of $(\bar{M}, \bar{g})$. Then the following assertions are equivalent:

(i) $S(T M)$ is parallel with respect to the induced connection $\nabla$;

(ii) $h^{*}$ vanishes identically on $M$;

(iii) $A_{N}$ vanishes identically on $M$.

Then it is easy to see that the following relations hold:

$$
\begin{gathered}
B(X, \xi)=0 \quad \forall X \in \Gamma\left(\left.T M\right|_{u}\right), \\
B(X, Y)=g\left(\stackrel{*}{\left.A_{\xi} X, Y\right),}\right. \\
\stackrel{*}{A_{\xi}} \xi=0 .
\end{gathered}
$$

3. Pseudoinverse of a degenerate metric. We start this section by the construction of the isomorphisms \# and $b$ with respect to the noninvertible induced metric $g$.

First, we consider on $M$ the 1-form $\eta$ defined by

$$
\eta(\cdot)=\bar{g}(N, \cdot),
$$

where $N$ is given by (2.1). Let $P$ be the projection morphism of $\Gamma(T M)$ on $\Gamma(S T M)$ in (2.2). We have, for all $X \in \Gamma(T M)$,

$$
X=P X+\eta(X) \xi
$$


and from (2.1) and (3.1),

$$
\eta(X)=0 \Longleftrightarrow X \in \Gamma(S T M) .
$$

Now, we define $b$ by

$$
\begin{aligned}
b: \Gamma(T M) & \longrightarrow \Gamma\left(T^{*} M\right), \\
X & \longmapsto X^{b}
\end{aligned}
$$

such that

$$
X^{b}=g(P X, \cdot)+\eta(X) \eta(\cdot)
$$

It is obvious that $b$ is linear.

As $T M$ and $T^{*} M$ have the same finite-constant rank, it suffices to show that $b$ is injective, that is, $X^{b}=0 \Leftrightarrow X=0$, to get the bijection of $b$. Consider $X \in \Gamma(T M)$ and assume $X^{b}=0$. Then

$$
X^{b}(Y)=0 \quad \forall Y \in \Gamma(T M) \Longleftrightarrow g(P X, Y)+\eta(X) \eta(Y)=0, \forall Y \in \Gamma(T M) .
$$

In particular, for any $Y \in \Gamma(S(T M))$, we have

$$
g(P X, Y)=0
$$

since $\eta(Y)=0$.

As $\Gamma(S(T M))$ is nondegenerate and $P X \in \Gamma(S T M)$, we have

$$
P X=0
$$

Therefore, $X$ satisfies

$$
\eta(X) \eta(Y)=0 \quad \forall Y \in \Gamma(T M) .
$$

In particular, if $Y=\xi, \eta(X)=0$. Hence, $X=P X+\eta(X) \xi=0$ and $b$ is injective. We conclude that the so-defined $b$ is an isomorphism of $\Gamma(T M)$ on $\Gamma\left(T^{*} M\right)$. We denote by \# its inverse isomorphism.

Note that the above construction generalizes the one of nondegenerate case for, in the latter case, $S(T M)$ coincides with $T M$. As a consequence, the 1 -form $\eta$ vanishes identically and $P$ becomes the identity map on $T M$.

We now define the associate metric $\tilde{g}$ of $g$. Put

$$
\tilde{\mathfrak{g}}(X, Y)=X^{b}(Y)
$$

It is obvious that $\tilde{g}$ is bilinear in $X$ and $Y$. Furthermore, from (3.5), we have that $X^{b}(Y)=Y^{b}(X)$, then $\tilde{g}$ is symmetric. Its inverse $\tilde{g}^{-1}$, which we denote by $g^{[\cdot, \cdot]}$, will be called the pseudoinverse of the degenerate metric $g$ on $M$. 
Note that in the case where $g$ is nondegenerate, the associate metric $\tilde{g}$ coincides with $g$ and the pseudoinverse $g^{[\cdot, \cdot]}$ coincides with the dual $g^{*}\left(\right.$ or $\left.g^{-1}\right)$ of $g$.

In what follows, we use the following range of indices:

$$
i, j=1, \ldots, n, \quad \alpha, \beta, \gamma=0,1, \ldots, n, \quad A, B, C, \ldots=0,1, \ldots, n+1 .
$$

Now, consider a local coordinate system $\left(x^{0}, \ldots, x^{n+1}\right)$ adapted to decompositions (2.2) and (2.3), that is,

$$
\left(X_{i}:=\frac{\partial}{\partial x^{i}}, \xi:=\frac{\partial}{\partial x^{0}}, N:=\frac{\partial}{\partial x^{n+1}}\right)
$$

is a local quasiorthogonal basis of $T \bar{M}_{\mid M}$ such that

$$
\begin{gathered}
T M^{\perp}=\operatorname{Span}\{\xi\}, \quad S(T M)=\operatorname{Span}\left\{X_{1}, \ldots, X_{n}\right\}, \\
\operatorname{tr}(T M)=\operatorname{Span}\{N\} .
\end{gathered}
$$

Using (3.1), (3.2), (3.3), and (3.5), we have a standard computation that gives

$$
\begin{gathered}
\left(\frac{\partial}{\partial x^{0}}\right)^{b}\left(\frac{\partial}{\partial x^{0}}\right)=0+\eta\left(\frac{\partial}{\partial x^{0}}\right) \eta\left(\frac{\partial}{\partial x^{0}}\right)=1=d x^{0}\left(\frac{\partial}{\partial x^{0}}\right), \\
\left(\frac{\partial}{\partial x^{0}}\right)^{b}\left(X_{i}\right)=g\left(P \xi, X_{i}\right)+\eta(\xi) \eta\left(X_{i}\right)=0=d x^{0}\left(\frac{\partial}{\partial x^{i}}\right),
\end{gathered}
$$

so

$$
\left(\frac{\partial}{\partial x^{0}}\right)^{b}=d x^{0}
$$

and the first terms in (3.14) are $\tilde{g}_{00}$ and $\tilde{g}_{0 i}$, respectively. Hence we have

$$
\tilde{\mathfrak{g}}_{00}=1, \quad \tilde{\mathfrak{g}}_{0 i}=\tilde{\mathfrak{g}}_{i 0}=0, \quad i=1, \ldots, n .
$$

We also have

$$
\left(\frac{\partial}{\partial x^{i}}\right)^{b}\left(\frac{\partial}{\partial x^{k}}\right)=g\left(P X_{i}, X_{k}\right)+\eta\left(X_{i}\right) \eta\left(X_{k}\right)=g_{i k} .
$$

Consequently,

$$
\tilde{g}_{i j}=g_{i j}, \quad i, j=1, \ldots, n .
$$


Thus, with respect to the quasiorthonormal basis $\left(\xi, X_{1}, \ldots, X_{n}, N\right)$, the matrices of $\tilde{g}$ and $g^{[\cdot, \cdot]}$ are given by

$$
\begin{aligned}
\tilde{g} & =\left(\begin{array}{cccc}
1 & 0 & \cdots & 0 \\
0 & & & \\
\vdots & & g_{i j} & \\
0 & & &
\end{array}\right), \\
g^{[\cdot, \cdot]} & =\left(\begin{array}{cccc}
1 & 0 & \cdots & 0 \\
0 & & & \\
\vdots & & \left(g_{i j}\right)^{-1} &
\end{array}\right) .
\end{aligned}
$$

We have

$$
\mathfrak{g}^{[\cdot]} \cdot \mathfrak{g}=\mathfrak{g} \cdot \mathfrak{g}^{[\cdot]}=\left(\begin{array}{cccc}
0 & 0 & \cdots & 0 \\
0 & 1 & & \vdots \\
\vdots & & \ddots & \\
0 & & \cdots & 1
\end{array}\right) \text {, }
$$

and this justifies the terminology pseudoinverse.

By (3.17) and (3.18), we have

$$
\left(\frac{\partial}{\partial x^{i}}\right)^{b}\left(\frac{\partial}{\partial x^{k}}\right)=g_{i k}=\tilde{g}_{i j} d x^{j}\left(\frac{\partial}{\partial x^{k}}\right), \quad i, j=1, \ldots, n
$$

Hence

$$
\left(\frac{\partial}{\partial x^{i}}\right)^{b}=\tilde{g}_{i j} d x^{j}
$$

Taking into account (3.15) and (3.22), we have

$$
d x^{\beta}=g^{[\alpha \beta]}\left(\frac{\partial}{\partial x^{\alpha}}\right)^{b}, \quad \alpha, \beta=0, \ldots, n
$$

From (3.19) and (3.23), remark that if $\rho$ is an endomorphism of TM (resp., a bilinear form on $T M$ ), its trace with respect to $g$ is given by

$$
\begin{aligned}
& \operatorname{trace}_{g} \rho=\sum_{\alpha=0}^{n} \tilde{g}\left(\rho\left(X_{\alpha}\right), X_{\alpha}\right), \\
& \operatorname{trace}_{g} \rho=g^{[\alpha \beta]} \rho_{\alpha \beta},
\end{aligned}
$$

respectively. 
We use (3.19) and (3.23) to deduce that

$$
\begin{gathered}
\tilde{\mathcal{g}}(\xi, \xi)=1, \\
\tilde{\mathfrak{g}}(X, Y)=\mathcal{g}(X, Y) \quad \forall X, Y \in S(T M), \\
\tilde{\mathcal{g}}(\xi, X)=\eta(X) \quad \forall X \in T M .
\end{gathered}
$$

Now, let $f: M \rightarrow \mathbb{R}$ be a smooth function on $M$. One defines intrinsically the gradient of $f$ by $\operatorname{grad}^{g} f=(d f)^{\#}$. But

$$
d f=\frac{\partial f}{\partial x^{\alpha}} d x^{\alpha} \Longrightarrow(d f)^{\#}=\frac{\partial f}{\partial x^{\alpha}}\left(d x^{\alpha}\right)^{\#} .
$$

Hence, from (3.23), we infer that

$$
\begin{gathered}
\operatorname{grad}^{g} f=\frac{\partial f}{\partial x^{\alpha}} g^{[\alpha \beta]}\left(\left(\frac{\partial}{\partial x^{\beta}}\right)^{b}\right)=\frac{\partial f}{\partial x^{\alpha}} g^{[\alpha \beta]} \frac{\partial}{\partial x^{\beta}}, \\
\operatorname{grad}^{g} f=g^{[\alpha \beta]} \frac{\partial f}{\partial x^{\alpha}} \frac{\partial}{\partial x^{\beta}} .
\end{gathered}
$$

Let $X$ be a smooth vector field defined on $U \subset M$. The divergence $\operatorname{div}^{g} X$ of $X$ with respect to the degenerate metric $g$ is intrinsically defined by

$$
\operatorname{div}^{g} X=-\sum_{\alpha=0}^{n} \varepsilon_{\alpha} X_{\alpha}^{b}\left(\nabla_{X_{\alpha}} X\right) .
$$

Therefore, from (3.10), we have

$$
\operatorname{div}^{\mathfrak{g}} X=-\sum_{\alpha=0}^{n} \varepsilon_{\alpha} \tilde{\mathfrak{g}}\left(\nabla_{X_{\alpha}} X, X_{\alpha}\right)
$$

with $\varepsilon_{0}=1$.

In summary, we have proved the following proposition.

Proposition 3.1. Let $(M, g, S(T M))$ be a lightlike hypersurface of a semiRiemannian $(n+2)$-dimensional manifold $(\bar{M}, \bar{g})$. There exists an associate metric $\tilde{g}$ and a pseudoinverse $g^{[\cdot]}$ of $g$ on $M$ such that locally on $u \subset M$, the following holds:

(i) for any smooth function $f: U \subset M \rightarrow \mathbb{R}$,

$$
\operatorname{grad}^{g} f=g^{[\alpha \beta]} f_{\alpha} \partial_{\beta},
$$

where $f_{\alpha}=\partial f / \partial x^{\alpha}, \partial_{\beta}=\partial / \partial x^{\beta}, \alpha, \beta=0, \ldots, n$;

(ii) for any vector field $X$ on $u \subset M$,

$$
\operatorname{div}^{\mathfrak{g}} X=-\sum_{\alpha=0}^{n} \varepsilon_{\alpha} \tilde{\mathfrak{g}}\left(\nabla_{X_{\alpha}} X, X_{\alpha}\right), \quad \varepsilon_{0}=1 ;
$$


(iii) for a smooth function $f$ defined on $U \subset M$,

$$
\Delta^{g} f=-\sum_{\alpha=0}^{n} \varepsilon_{\alpha} \tilde{\mathfrak{g}}\left(\nabla_{X_{\alpha}} \operatorname{grad}^{g} f, X_{\alpha}\right) .
$$

Here $\Delta^{g}$ is the d'Alembertian with respect to $g$ on $u$.

Note that if the section $\xi$ is globally defined on $M$, then our result is also global.

4. Compatibility of $\tilde{g}$ with respect to the connection $\nabla$ induced by $g$. It is well known that the induced metric $g$ is not compatible with the induced connection $\nabla$ in general, and this compatibility arises if and only if the lightlike hypersurface $M$ is totally geodesic in $\bar{M}$. This section deals with the compatibility of the associate metric $\tilde{g}$ with respect to $\nabla$.

For $X, Y, Z \in \Gamma(T M)$, we have

$$
\begin{aligned}
\left(\nabla_{X} \tilde{\mathfrak{g}}\right) & (Y, Z) \\
= & X \cdot \tilde{g}(Y, Z)-\tilde{g}\left(\nabla_{X} Y, Z\right)-\tilde{g}\left(Y, \nabla_{X} Z\right) \\
= & X \cdot\{\tilde{\mathfrak{g}}(P Y, P Z)+\tilde{\mathfrak{g}}(P Y, \eta(Z) \xi)+\tilde{\mathfrak{g}}(\eta(Y) \xi, P Z)+\eta(Y) \eta(Z) \tilde{\mathfrak{g}}(\xi, \xi)\} \\
& -\tilde{g}\left(\nabla_{X}(P Y+\eta(Y) \xi), P Z+\eta(Z) \xi\right)-\tilde{g}\left(P Y+\eta(Y) \xi, \nabla_{X}(P Z+\eta(Z) \xi)\right) \\
= & X \cdot\{g(P Y, P Z)+\eta(Z) \eta(P Y)+\eta(Y) \eta(P Z)+\eta(Y) \eta(Z)\} \\
& -\tilde{g}\left(\nabla_{X} P Y+\nabla_{X}(\eta(Y) \xi), P Z\right)-\tilde{g}\left(\nabla_{X} P Y+\nabla_{X}(\eta(Y) \xi), \eta(Z) \xi\right) \\
& -\tilde{g}\left(P Y, \nabla_{X} P Z+\nabla_{X}(\eta(Z) \xi)\right)-\tilde{g}\left(\eta(Y) \xi, \nabla_{X} P Z+\nabla_{X}(\eta(Z) \xi)\right) \\
= & X \cdot\{g(P Y, P Z)+\eta(Y) \eta(Z)\}-\tilde{g}\left(\nabla_{X} P Y, P Z\right)-\tilde{g}\left(\nabla_{X}(\eta(Y) \xi), P Z\right) \\
& -\tilde{g}\left(\nabla_{X} P Y, \eta(Z) \xi\right)-\tilde{g}\left(\nabla_{X}(\eta(Y) \xi), \eta(Z) \xi\right)-\tilde{g}\left(P Y, \nabla_{X} P Z\right) \\
& -\tilde{g}\left(P Y, \nabla_{X}(\eta(Z) \xi)\right)-\tilde{g}\left(\eta(Y) \xi, \nabla_{X} P Z\right)-\tilde{g}\left(\eta(Y) \xi, \nabla_{X}(\eta(Z) \xi)\right) .
\end{aligned}
$$

Now use (2.9), (2.11), and (3.10) to obtain

$$
\begin{aligned}
\left(\nabla_{X} \tilde{\mathfrak{g}}\right)(Y, Z)= & X \cdot\{g(P Y, P Z)\}+\eta(Y) X \cdot \eta(Z)+\eta(Z) X \cdot \eta(Y) \\
& -\tilde{\mathfrak{g}}\left(\stackrel{*}{\nabla}_{X} P Y, P Z\right)-\tilde{\mathfrak{g}}(C(X, P Y) \xi, P Z) \\
& -g\left(\stackrel{*}{\nabla}_{X} P Y, P Z\right)-\eta(P Z) C(X, P Y)-(X \cdot \eta(Y)) \eta(P Z) \\
& -\eta(Y) \tilde{\mathfrak{g}}\left(-A_{\xi}^{*}, P Z\right)-\eta(Y) \tilde{\mathfrak{g}}(-\tau(X) \xi, P Z) \\
& -\eta(Z) \eta\left(\stackrel{*}{\nabla}_{X} P Y\right)-\eta(Z) C(X, P Y)-\eta(Z) X \cdot \eta(Y)
\end{aligned}
$$




$$
\begin{aligned}
& -\eta(Y) \eta(Z) \tilde{g}\left(-\stackrel{*}{A}_{\xi} X, \xi\right)-\eta(Y) \eta(Z) \tilde{g}(-\tau(X) \xi, \xi) \\
& -g\left(P Y,{ }^{\nabla}{ }_{X} P Z\right)-C(X, P Z) \eta(P Y)-\eta(P Y) X \cdot \eta(Z) \\
& -\eta(Z) \tilde{g}\left(P Y, \stackrel{*}{A}_{\xi} X\right)-\eta(Z) \tilde{g}(-P Y,-\tau(X) \xi) \\
& -\eta(Y) \eta\left(\stackrel{*}{\nabla}_{X} P Z\right)-\eta(Y) C(X, P Z)-\eta(Y) X \cdot(\eta(Z)) \\
& -\eta(Z) \eta(Y) \tilde{g}\left(\xi,-\stackrel{*}{A}_{\xi} X\right)-\eta(Z) \eta(Y) \tilde{g}(\xi,-\tau(X) \xi) .
\end{aligned}
$$

But from (3.3), we know that $\eta(X)=0$ if and only if $X \in \Gamma(S(T M))$. Using (2.16) and (3.2) leads to

$$
\begin{aligned}
\left(\nabla_{X} \tilde{g}\right)(Y, Z)= & X \cdot\{g(P Y, P Z)\}+\eta(Y) X \cdot \eta(Z)+\eta(Z) X \cdot \eta(Y) \\
& -\tilde{g}\left(\stackrel{*}{\nabla}_{X} P Y, P Z\right)+\eta(Y) B(X, P Z)-\eta(Z) C(X, P Y) \\
& -\eta(Z) X \cdot(\eta(Y))+\tau(X) \eta(Y) \eta(Z)-g\left(P Y, \stackrel{*}{\nabla}_{X} P Z\right) \\
& +\eta(Z) B(X, P Y)-\eta(Y) C(X, P Z)-\eta(Y) X \cdot(\eta(Z)) \\
& +\tau(X) \eta(Y) \eta(Z) .
\end{aligned}
$$

The above expression reduces to

$$
\begin{aligned}
\left(\nabla_{X} \tilde{g}\right)(Y, Z)= & \left\{X \cdot g(P Y, P Z)-g\left(\stackrel{*}{\nabla}_{X} P Y, P Z\right)-g\left(P Y, \stackrel{*}{\nabla}_{X} P Z\right)\right\} \\
& +\eta(Y) B(X, P Z)+\eta(Z) B(X, P Y)-\eta(Z) C(X, P Y) \\
& -\eta(Y) C(X, P Z)+2 \tau(X) \eta(Y) \eta(Z) .
\end{aligned}
$$

Now, recall that since the connection $\stackrel{*}{\nabla}$ is compatible with $g$, the expression between brackets is zero, and therefore we have

$$
\begin{aligned}
\left(\nabla_{X} \tilde{g}\right)(Y, Z)= & \eta(Y)(B(X, P Z)-C(X, P Z)) \\
& +\eta(Z)(B(X, P Y)-C(X, P Y)) \\
& +2 \tau(X) \eta(Y) \eta(Z) \quad \forall X, Y, Z \in \Gamma(T M) .
\end{aligned}
$$

Then we get the following theorem.

THEOREM 4.1. Let $(M, g, S(T M))$ be a lightlike hypersurface of a semiRiemannian manifold $(\bar{M}, \bar{g})$. The induced connection $\nabla$ by $g$ on $M$ is compatible with the associate metric $\tilde{g}$ of $g$ if and only if, for all $X, Z \in \Gamma(T M)$,

$$
B(X, P Z)=C(X, P Z), \quad \tau(X)=0 .
$$


Proof. It is well known that $\nabla$ is compatible with $\tilde{g}$ if and only if $\left(\nabla_{X} \tilde{\mathfrak{g}}\right)(Y, Z)$ $=0$ for all $X, Y, Z \in \Gamma(T M)$. Putting $Y=Z=\xi$ in (4.5) gives $\tau(X)=0$ for all $X \in \Gamma(T M)$, and setting $Y=\xi$ yields $B(X, P Z)=C(X, P Z)$ for all $X, Z \in \Gamma(T M)$.

We derive from (4.5) the main covariant derivative formula

$$
\begin{aligned}
X \cdot \tilde{g}(X, Z)= & \tilde{g}\left(\nabla_{X} Y, Z\right)+\tilde{g}\left(Y, \nabla_{X} Z\right)+\eta(Y)(B(X, P Z)-C(X, P Z)) \\
& +\eta(Z)(B(X, P Y)-C(X, P Y))+2 \tau(X) \eta(Y) \eta(Z) .
\end{aligned}
$$

We discuss here the effect of the change of the screen distribution on the compatibility of $\tilde{g}$ with $g$. The first equation in (4.6) indicates that only some privileged changes in the screen distribution maintain the compatibility property of the associate metric $\tilde{g}$ with $g$. For two screen distributions $S(T M)$ and $S(T M)^{\prime}$ on $(M, g)$, with two orthogonal frame fields $\left(W_{i}\right)_{i=1, \ldots, n}$ and $\left(W_{i}^{\prime}\right)_{i=1, \ldots, n}$, respectively, the relationship between the second fundamental forms $C$ and $C^{\prime}$ of the screen distributions $S(T M)$ and $S(T M)^{\prime}$, respectively, is given by (see [4])

$$
C^{\prime}(X, P Y)=C(X, P Y)-\frac{1}{2}\langle W, W\rangle B(X, Y)+g\left(\nabla_{X} P Y, W\right)
$$

where $W=\sum_{i=1}^{i=n} c_{i} W_{i}$ is the characteristic vector field of the screen change.

Therefore, a change in screen distribution leaves the compatibility of $\tilde{g}$ with $g$ invariant if and only if

$$
\omega\left(\nabla_{X} P Y-\frac{1}{2} B(X, Y) W\right)=0 \quad \forall X, Y \in \Gamma(T M)
$$

where $\omega$ is the dual 1 -form of $W$ defined by $\omega(\cdot)=g(W, \cdot)$.

From (3.1) and (3.10), if $\tilde{g}$ is associated to $g$ on $S(T M)$ and $\tilde{g}^{\prime}$ is associated to $g$ on $S(T M)^{\prime}$, we have

$$
\tilde{\mathfrak{g}}^{\prime}(X, Y)=\tilde{g}(X, Y)+D(X, Y),
$$

where $D$ is the bilinear form defined by

$$
D(X, Y)=\eta(X) \omega(Y)+\eta(Y) \omega(X)+\omega(X) \omega(Y) .
$$

Before starting the next section, it is important to stress that the 1 -form $\tau$ of (4.6), defined in (2.6) and (2.8), depends on the normalization of the rankone subbundle $\operatorname{Rad}(T M)$, that is, on the choice of $\xi \in \Gamma\left(\left.T M^{\perp}\right|_{\ell}\right)$. It has been proved in [11, page 99] that there exists a pair (a normalization) $\{\xi, N\}$ on $U$ such that the corresponding 1 -form $\tau$ vanishes identically. 
From now on we use this normalization. Then, relations (2.6) and (2.14) become

$$
\begin{aligned}
& \bar{\nabla}_{X} N=-A_{N} X, \\
& \nabla_{X} \xi=-A_{\xi} X,
\end{aligned}
$$

for all $X \in \Gamma(T M)$

5. Laplacian of lightlike hypersurfaces endowed with parallel screen distribution. Let $\left(M^{n+1}, g, S(T M)\right)$ be a lightlike hypersurface of the Lorentzian space $\mathbb{R}_{1}^{n+2}$. The metric on $\mathbb{R}_{1}^{n+2}$ and the position vector field of $M^{n+1}$ will be denoted by $\langle\cdot, \cdot\rangle$ and $x$, respectively.

We have

$$
\begin{aligned}
& T M=\operatorname{Rad}(T M) \perp S(T M)=T M^{\perp} \perp S(T M), \\
& \left.T \mathbb{R}_{1}^{n+2}\right|_{M}=(\operatorname{Rad}(T M) \oplus \operatorname{tr}(T M)) \perp S(T M),
\end{aligned}
$$

and $\left(X_{0}:=\xi, N, X_{1}, \ldots, X_{n}\right)$ is a local quasiorthonormal frame field on $\mathbb{R}_{1}^{n+2}$ such that at each point $x \in M,\left(X_{1}, \ldots, X_{n}\right)$ represents a basis of $S\left(T_{x} M\right)$. As the plane spanned by the pair $\left\{\xi_{x}, N_{x}\right\}$ is a hyperbolic plane at any $x \in M$ and $\mathbb{R}_{1}^{n+2}$ has index $q=1$, it follows that the screen distribution on $M \subset \mathbb{R}_{1}^{n+2}$ is Riemannian, that is, the induced metric on $S(T M)$ is positive definite. As a consequence, the signature $\varepsilon_{k}$ of $X_{k}, k=1, \ldots, n$, is $\varepsilon_{k}=1$.

Define on $M$ the smooth functions

$$
f_{0}(x)=\langle x, N\rangle, \quad f_{n+1}(x)=\langle x, \xi\rangle, \quad f_{k}(x)=\left\langle x, X_{k}\right\rangle, \quad k=1, \ldots, n,
$$

where $x$ stands for the position vector field of $M$ in $\mathbb{R}_{1}^{n+2}$. We have

$$
U_{A}:=\operatorname{grad}^{g} f_{A}=g^{[\alpha \gamma]} f_{A ; \alpha} X_{\gamma},
$$

where $f_{A ; \alpha}:=X_{\alpha} \cdot f_{A}$.

The position vector field $x$ can be written in $\mathbb{R}_{1}^{n+2}$ as

$$
x=\left(\langle x, N\rangle,\langle x, \xi\rangle, \varepsilon_{1}\left\langle x, X_{1}\right\rangle, \ldots, \varepsilon_{n}\left\langle x, X_{n}\right\rangle\right)
$$

with respect to $\left(\xi, N, X_{1}, \ldots, X_{n}\right)$. As $\varepsilon_{k}=1, k=1, \ldots, n$, we have

$$
x=\left(f_{0}(x), f_{n+1}(x), f_{1}(x), \ldots, f_{n}(x)\right) .
$$

Let $\Delta^{g}$ denote the Laplace operator on functions with respect to the degenerate metric $g$. Then

$$
\Delta^{g} x=\left(\Delta^{g} f_{0}(x), \Delta^{g} f_{N}(x), \Delta^{g} f_{1}(x), \ldots, \Delta^{g} f_{n+1}(x)\right) .
$$


But

$$
\Delta^{g} f_{A}=-\sum_{\alpha=0}^{n} \varepsilon_{\alpha} \tilde{g}\left(\nabla_{X_{\alpha}} U_{A}, X_{\alpha}\right) .
$$

According to the above remark on the signature and (2.1), we have here $\varepsilon_{\alpha}=1$ for all $\alpha$.

Using (4.7) yields

$$
\begin{aligned}
\tilde{g}\left(\nabla_{X_{\alpha}} U_{A}, X_{\alpha}\right)= & X_{\alpha} \cdot \tilde{g}\left(U_{A}, X_{\alpha}\right)-\tilde{g}\left(U_{A}, \nabla_{X_{\alpha}} X_{\alpha}\right) \\
& -\eta\left(U_{A}\right)\left(B\left(X_{\alpha}, P X_{\alpha}\right)-C\left(X_{\alpha}, P X_{\alpha}\right)\right) \\
& -\eta\left(X_{\alpha}\right)\left(B\left(X_{\alpha}, P U_{A}\right)-C\left(X_{\alpha}, P U_{A}\right)\right) \\
& -2 \tau\left(X_{\alpha}\right) \eta\left(X_{\alpha}\right) \eta\left(U_{A}\right) .
\end{aligned}
$$

In this section, we assume $S(T M)$ parallel. From Theorem 2.3 and (2.11), we have $C=0$ and $A_{N}=0$ on $M$. Moreover, we can write, without loss of generality, $\nabla_{X} X_{i}=0$ for all $X \in \Gamma(T M)$, where the $X_{i}$ 's are vectors of a local basis of $\Gamma(S(T M))$. Finally, as $\tau=0$, we have

$$
\begin{aligned}
\tilde{g}\left(\nabla_{X_{\alpha}} U_{A}, X_{\alpha}\right)= & X_{\alpha} \cdot \tilde{g}\left(U_{A}, X_{\alpha}\right)-\tilde{g}\left(U_{A}, \nabla_{X_{\alpha}} X_{\alpha}\right) \\
& -\eta\left(U_{A}\right) B\left(X_{\alpha}, P X_{\alpha}\right)-\eta\left(X_{\alpha}\right) B\left(X_{\alpha}, P U_{A}\right), \quad \alpha=0, \ldots, n .
\end{aligned}
$$

But

$$
\begin{aligned}
X_{\alpha} \cdot \tilde{g}\left(U_{A}, X_{\alpha}\right) & =X_{\alpha} \cdot\left\{\tilde{g}\left(g^{[\varepsilon \gamma]} f_{A ; \varepsilon} X_{\gamma}, X_{\alpha}\right)\right\} \\
& =X_{\alpha} \cdot\left\{f_{A ; \varepsilon} g^{[\varepsilon \gamma]} \tilde{g}_{\gamma \alpha}\right\}=X_{\alpha} \cdot\left\{\delta_{\alpha}^{\varepsilon} f_{A ; \varepsilon}\right\}
\end{aligned}
$$

Hence

$$
X_{\alpha} \cdot \tilde{g}\left(U_{A}, X_{\alpha}\right)=X_{\alpha} \cdot\left\{f_{A ; \alpha}\right\}=: f_{A ; \alpha \alpha} .
$$

Now we compute $f_{A ; \alpha}$ and $f_{A ; \alpha \alpha}$ for $A=0, \ldots, n+1$ and $\alpha=0, \ldots, n$ :

$$
f_{0}(x)=\langle x, N\rangle \Longrightarrow f_{0 ; \alpha}=X_{\alpha} \cdot\langle x, N\rangle=\left\langle\bar{\nabla}_{X_{\alpha}} x, N\right\rangle+\left\langle x, \bar{\nabla}_{X_{\alpha}} N\right\rangle .
$$

Note that as $\tau=0$ and $S(T M)$ is parallel, $N$ is a parallel vector field. Indeed, $\bar{\nabla}_{X} N=-A_{N} X+\tau(X) N$. It follows from (4.12) and Theorem 2.3 that

$$
\bar{\nabla}_{X} N=0 \quad \forall X \in \Gamma(T M) .
$$

Hence

$$
\begin{aligned}
f_{0 ; \alpha} & =\left\langle X_{\alpha}, N\right\rangle \\
f_{0 ; \alpha \alpha} & =X_{\alpha} \cdot\left\langle X_{\alpha}, N\right\rangle \\
& =\left\langle\bar{\nabla}_{X_{\alpha}} X_{\alpha}, N\right\rangle+\left\langle X_{\alpha}, \bar{\nabla}_{X_{\alpha}} N\right\rangle \\
& =\left\langle\nabla_{X_{\alpha}} X_{\alpha}+B\left(X_{\alpha}, X_{\alpha}\right) N, N\right\rangle .
\end{aligned}
$$


Thus

$$
\begin{gathered}
f_{0 ; \alpha \alpha}=\left\langle\nabla_{X_{\alpha}} X_{\alpha}, N\right\rangle \\
f_{n+1}(x)=\langle x, \xi\rangle \Longrightarrow f_{n+1 ; \alpha}=\left\langle X_{\alpha}, \xi\right\rangle+\left\langle x, \bar{\nabla}_{X_{\alpha}} \xi\right\rangle .
\end{gathered}
$$

Therefore,

$$
\begin{aligned}
f_{n+1 ; \alpha} & =\left\langle x, \bar{\nabla}_{X_{\alpha}} \xi\right\rangle \\
f_{n+1 ; \alpha \alpha} & =X_{\alpha} \cdot\left\langle x, \bar{\nabla}_{X_{\alpha}} \xi\right\rangle \\
& =\left\langle X_{\alpha}, \bar{\nabla}_{X_{\alpha}} \xi\right\rangle+\left\langle x, \bar{\nabla}_{X_{\alpha}} \bar{\nabla}_{X_{\alpha}} \xi\right\rangle \\
& =-B\left(X_{\alpha}, X_{\alpha}\right)+\left\langle x, \bar{\nabla}_{X_{\alpha}} \bar{\nabla}_{X_{\alpha}} \xi\right\rangle .
\end{aligned}
$$

But a straightforward computation leads to

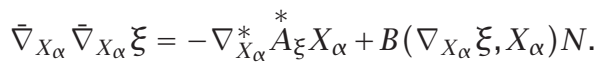

We also obtain

$$
\begin{aligned}
\left\langle-\nabla_{X_{\alpha}}^{*} \stackrel{*}{A}_{\xi} X_{\alpha}, X_{i}\right\rangle & =-X_{\alpha} \cdot\left\langle\stackrel{*}{A_{\xi}} X_{\alpha}, X_{i}\right\rangle+B\left(X_{\alpha}, \stackrel{*}{\nabla}_{X_{\alpha}} X_{i}\right) \\
& =-X_{\alpha} \cdot B\left(X_{\alpha}, X_{i}\right),
\end{aligned}
$$

where we have used

$$
\stackrel{*}{\nabla}_{X_{\alpha}} X_{i}=\nabla_{X_{\alpha}} X_{i}-C\left(X_{\alpha}, X_{i}\right)=\nabla_{X_{\alpha}} X_{i}=0 .
$$

Therefore,

$$
\begin{aligned}
\left\langle x, \bar{\nabla}_{X_{\alpha}} \bar{\nabla}_{X_{\alpha}} \xi\right\rangle & =B\left(\nabla_{X_{\alpha}} \xi, X_{\alpha}\right)\langle x, N\rangle-\sum_{i=1}^{n}\left\langle x, X_{i}\right\rangle X_{\alpha} \cdot B\left(X_{\alpha}, X_{i}\right) \\
& =-B\left({ }^{*} A_{\xi} X_{\alpha}, X_{\alpha}\right)\langle x, N\rangle-\sum_{i=1}^{n}\left\langle x, X_{i}\right\rangle X_{\alpha} \cdot B\left(X_{\alpha}, X_{i}\right),
\end{aligned}
$$

so

$$
\begin{aligned}
f_{n+1 ; \alpha \alpha}= & -B\left(X_{\alpha}, X_{\alpha}\right)-B\left(\stackrel{*}{A_{\xi}} X_{\alpha}, X_{\alpha}\right)\langle x, N\rangle \\
& -\sum_{i=1}^{n}\left\langle x, X_{i}\right\rangle X_{\alpha} \cdot B\left(X_{\alpha}, X_{i}\right), \\
f_{k}(x)= & \left\langle x, X_{k}\right\rangle \Rightarrow f_{k ; \alpha}=\left\langle X_{\alpha}, X_{k}\right\rangle+\left\langle x, \bar{\nabla}_{X_{\alpha}} X_{k}\right\rangle, \\
f_{k ; \alpha}= & \left\langle X_{\alpha}, X_{k}\right\rangle+\left\langle x, B\left(X_{\alpha}, X_{k}\right) N\right\rangle, \\
f_{k ; \alpha \alpha}= & \left\langle\bar{\nabla}_{X_{\alpha}} X_{\alpha}, X_{k}\right\rangle+\left\langle X_{\alpha}, \bar{\nabla}_{X_{\alpha}} X_{k}\right\rangle \\
& +X_{\alpha} \cdot B\left(X_{\alpha}, X_{k}\right)\langle x, N\rangle+B\left(X_{\alpha}, X_{k}\right)\left\langle X_{\alpha}, N\right\rangle .
\end{aligned}
$$


Moreover,

$$
\left\langle\bar{\nabla}_{X_{\alpha}} X_{\alpha}, X_{k}\right\rangle=\left\langle\nabla_{X_{\alpha}} X_{\alpha}+B\left(X_{\alpha}, X_{\alpha}\right) N, X_{k}\right\rangle=\left\langle\nabla_{X_{\alpha}} X_{\alpha}, X_{k}\right\rangle .
$$

But $\nabla_{X_{\alpha}} X_{\alpha}=0$ for all $\alpha$. Indeed, if $\alpha=0$, from (2.14) and (2.17), $\nabla_{\xi} \xi=0$. For $\alpha=i \neq 0, \nabla_{X_{i}} X_{i}=0$ from the condition of parallel screen distribution. So $\left\langle\bar{\nabla}_{X_{\alpha}} X_{\alpha}, X_{k}\right\rangle=0$. We also have that

$$
\left\langle X_{\alpha}, \bar{\nabla}_{X_{\alpha}} X_{k}\right\rangle=0, \quad B\left(X_{\alpha}, X_{k}\right)\left\langle X_{\alpha}, N\right\rangle=0
$$

for at least one of $B\left(X_{\alpha}, X_{k}\right)$ or $\left\langle X_{\alpha}, N\right\rangle$ being zero according to either $\alpha=0$ or $\alpha \neq 0$, so

$$
f_{k ; \alpha \alpha}=\left[X_{\alpha} \cdot B\left(X_{\alpha}, X_{k}\right)\right]\langle x, N\rangle .
$$

From $\nabla_{X_{\alpha}} X_{\alpha}=0$, the term $\tilde{g}\left(U_{A}, \nabla_{X_{\alpha}} X_{\alpha}\right)$ in expression (5.9) is zero.

Next we compute the term $\tilde{\mathfrak{g}}\left(\nabla_{X_{\alpha}} U_{A}, X_{\alpha}\right)$ in the left-hand side of (5.9) for $A=0, \ldots, n+1$ and $\alpha=0, \ldots, n$ :

$$
\tilde{\mathfrak{g}}\left(\nabla_{\xi} U_{0}, \xi\right)=f_{0 ; 00}-\tilde{\mathfrak{g}}\left(U_{0}, \nabla_{\xi} \xi\right)-\eta\left(U_{0} B(\xi, P \xi)\right)-\eta(\xi) B\left(\xi, P U_{0}\right)=0 .
$$

Hence

$$
\tilde{\mathfrak{g}}\left(\nabla_{\xi} U_{0}, \xi\right)=0
$$

Similar computations give the other terms as follows:

$$
\begin{aligned}
\tilde{\mathfrak{g}}\left(\nabla_{X_{k}} U_{0}, X_{k}\right)= & -B\left(X_{k}, X_{k}\right), \quad \tilde{g}\left(\nabla_{\xi} U_{k}, \xi\right)=0, \\
\tilde{\mathfrak{g}}\left(\nabla_{X_{l}} U_{k}, X_{l}\right)= & {\left[X_{l} \cdot B\left(X_{l}, X_{k}\right)\right]\langle x, N\rangle, \quad \tilde{\mathfrak{g}}\left(\nabla_{\xi} U_{n+1}, \xi\right)=0, } \\
\tilde{g}\left(\nabla_{X_{l}} U_{n+1}, X_{l}\right)= & -B\left(X_{l}, X_{l}\right)-B\left(\stackrel{*}{A_{\xi}} X_{l}, X_{l}\right)\langle x, N\rangle \\
& -\sum_{i=1}^{n}\left\langle x, X_{i}\right\rangle X_{l} \cdot B\left(X_{l}, X_{i}\right) .
\end{aligned}
$$

We will make use of the following lemma.

LEMmA 5.1. Let $(M, g, S(T M))$ be a lightlike hypersurface of $\mathbb{R}_{1}^{n+2}$. Set $B_{j k ; i}:=$ $X_{i} \cdot B\left(X_{j}, X_{k}\right)$. If $S(T M)$ is parallel and $\tau=0$, then $B_{j k ; i}$ is symmetric with respect to the indices $i, j$, and $k$.

Proof. Clearly, $B_{j k ; i}$ is symmetric with respect to $j$ and $k$. It suffices to show its symmetry with respect to $i$ and $j$. First,

$$
\left(\nabla_{X_{i}} B\right)\left(X_{j}, X_{k}\right)=X_{i} \cdot B\left(X_{j}, X_{k}\right)-B\left(\nabla_{X_{i}} X_{j}, X_{k}\right)-B\left(X_{j}, \nabla_{X_{i}} X_{k}\right) .
$$

Since $S(T M)$ is parallel, the two last terms of this equality are zero. Hence, for all $i, j, k=1, \ldots, n$,

$$
\left(\nabla_{X_{i}} B\right)\left(X_{j}, X_{k}\right)=X_{i} \cdot B\left(X_{j}, X_{k}\right)
$$


Next, Gauss-Codazzi equation gives

$$
\begin{aligned}
\bar{g}\left(\bar{R}\left(X_{i}, X_{j}\right) X_{k}, \xi\right)= & \left(\nabla_{X_{i}} B\right)\left(X_{j}, X_{k}\right)-\left(\nabla_{X_{j}} B\right)\left(X_{i}, X_{k}\right) \\
& +B\left(X_{j}, X_{k}\right) \tau\left(X_{i}\right)-B\left(X_{i}, X_{k}\right) \tau\left(X_{j}\right) .
\end{aligned}
$$

The ambient space is $\mathbb{L}^{n+2}:=\mathbb{R}_{1}^{n+2}$, hence $\bar{R}=0$. As $\tau=0$, we get

$$
\left(\nabla_{X_{i}} B\right)\left(X_{j}, X_{k}\right)=\left(\nabla_{X_{j}} B\right)\left(X_{i}, X_{k}\right)
$$

that is,

$$
X_{i} \cdot B\left(X_{j}, X_{k}\right)=X_{j} \cdot B\left(X_{i}, X_{k}\right),
$$

which shows that $B_{j k ; i}$ is symmetric with respect to $i$ and $j$. This achieves the proof.

Now we are in a position to compute each term $\Delta^{g} f_{A}$ in (5.7) for $A=0, \ldots, n+$ 1. We have

$$
\begin{aligned}
\sum_{\alpha=0}^{n} \varepsilon_{\alpha} \tilde{g}\left(\nabla_{X_{\alpha}} U_{0}, X_{\alpha}\right) & =\tilde{g}\left(\nabla_{\xi} U_{0}, \xi\right)+\sum_{k=1}^{n} \varepsilon_{k} \tilde{\mathfrak{g}}\left(\nabla_{X_{k}} U_{0}, X_{k}\right) \\
& =-\sum_{k=1}^{n} B\left(X_{k}, X_{k}\right) .
\end{aligned}
$$

Hence, using (2.15), we infer that

$$
\sum_{\alpha=0}^{n} \varepsilon_{\alpha} \tilde{\mathcal{g}}\left(\nabla_{X_{\alpha}} U_{0}, X_{\alpha}\right)=-\sum_{\alpha=0}^{n} B\left(X_{\alpha}, X_{\alpha}\right) .
$$

We also compute similar sums for $U_{n+1}$ and $U_{k}$ :

$$
\begin{aligned}
\sum_{\alpha=0}^{n} \varepsilon_{\alpha} \tilde{g}\left(\nabla_{X_{\alpha}} U_{n+1}, X_{\alpha}\right)= & \tilde{g}\left(\nabla_{\xi} U_{n+1}, \xi\right)+\sum_{k=1}^{n} \varepsilon_{k} \tilde{g}\left(\nabla_{X_{k}} U_{n+1}, X_{k}\right) \\
= & -\sum_{k=1}^{n} B\left(X_{k}, B\left(X_{k}\right)\right)-\left[\sum_{k=1}^{n} B\left(\stackrel{*}{A}_{\xi} X_{k}, X_{k}\right)\right]\langle x, N\rangle \\
& -\sum_{k=1}^{n} \sum_{i=1}^{n}\left\langle x, X_{i}\right\rangle X_{k} \cdot B\left(X_{k}, X_{i}\right) .
\end{aligned}
$$

By using Lemma 5.1 and (2.15), we obtain

$$
\begin{aligned}
\sum_{\alpha=0}^{n} \varepsilon_{\alpha} \tilde{g}\left(\nabla_{X_{\alpha}} U_{n+1}, X_{\alpha}\right)= & -\sum_{k=1}^{n} B\left(X_{k}, X_{k}\right)-\left[\sum_{k=1}^{n} B\left(\stackrel{*}{A_{\xi}} X_{k}, X_{k}\right)\right]\langle x, N\rangle \\
& -\sum_{i=1}^{n}\left[X_{i} \cdot \sum_{\alpha=0}^{n} B\left(X_{\alpha}, X_{\alpha}\right)\right]\left\langle x, X_{i}\right\rangle .
\end{aligned}
$$


Finally,

$$
\begin{aligned}
\sum_{\alpha=0}^{n} \varepsilon_{\alpha} \tilde{\mathfrak{g}}\left(\nabla_{X_{\alpha}} U_{k}, X_{\alpha}\right) & =\tilde{g}\left(\nabla_{\xi} U_{k}, \xi\right)+\sum_{l=1}^{n} \varepsilon_{l} \tilde{\mathfrak{g}}\left(\nabla_{X_{l}} U_{l}, X_{k}\right) \\
& =\sum_{l=1}^{n} X_{l} \cdot B\left(X_{l}, X_{k}\right)\langle x, N\rangle,
\end{aligned}
$$

and using Lemma 5.1 and (2.15), we also obtain

$$
\sum_{\alpha=0}^{n} \varepsilon_{\alpha} \tilde{\mathfrak{g}}\left(\nabla_{X_{\alpha}} U_{k}, X_{\alpha}\right)=\left[X_{k} \cdot \sum_{\alpha=0}^{n} B\left(X_{\alpha}, X_{\alpha}\right)\right]\langle x, N\rangle .
$$

Put

$$
H=\frac{1}{n+1}\left[\sum_{\alpha=0}^{n} B\left(X_{\alpha}, X_{\alpha}\right)\right](\xi+N)
$$

to obtain

$$
\begin{aligned}
\Delta^{g} x= & (n+1) H-\sum_{k=1}^{n}\langle x, N\rangle X_{k} \cdot\langle(n+1) H, N\rangle X_{k} \\
& +\left\{\sum_{\alpha=0}^{n} B\left(A_{\xi}^{*} X_{\alpha}, X_{\alpha}\right)\langle x, N\rangle-\sum_{i=1}^{n} X_{i} \cdot \sum_{\alpha=0}^{n} B\left(X_{\alpha}, X_{\alpha}\right)\left\langle x, X_{i}\right\rangle\right\} N .
\end{aligned}
$$

If we now put

$$
K=\left[\sum_{\alpha=0}^{n} B\left(X_{\alpha}, X_{\alpha}\right)\right]
$$

then

$$
\begin{aligned}
\Delta^{g} x= & (n+1) H-\sum_{k=1}^{n}\left(\langle x, N\rangle X_{k} \cdot K\right) X_{k} \\
& +\left\{\sum_{\alpha=0}^{n} B\left(\stackrel{*}{A}_{\xi} X_{\alpha}, X_{\alpha}\right)\langle x, N\rangle-\sum_{k=1}^{n}\left\langle x, X_{k}\right\rangle X_{k} \cdot K\right\} N .
\end{aligned}
$$

Thus, we have established the following theorem.

THEOREM 5.2. Let $(M, g, S(T M))$ be a lightlike hypersurface of the Lorentzian space $\mathbb{L}^{n+2}:=\mathbb{R}_{1}^{n+2}$, endowed with a parallel screen distribution $S(T M)$ and with symmetric Ricci tensor. Then its Laplacian with respect to the degenerate 
metric $g$ is given by

$$
\begin{aligned}
\Delta^{g} x= & (n+1) H-\sum_{k=1}^{n}\left(\langle x, N\rangle X_{k} \cdot K\right) X_{k} \\
& +\left\{\sum_{\alpha=0}^{n} B\left({ }^{*}{ }_{\xi} X_{\alpha}, X_{\alpha}\right)\langle x, N\rangle-\sum_{k=1}^{n}\left\langle x, X_{k}\right\rangle X_{k} \cdot K\right\} N .
\end{aligned}
$$

Definition 5.3. On the lightlike hypersurface $(M, g, S(T M))$, the smooth function $\sigma$ defined by

$$
\sigma=\frac{1}{\sqrt{2}(n+1)} K, \quad K=\sum_{\alpha}^{n} B\left(X_{\alpha}, X_{\alpha}\right),
$$

is called the mean curvature function of $M$.

6. An application. It is well known that minimal immersions of smooth manifolds $M$ into Euclidean spheres are those immersions whose coordinate functions in the ambient Euclidian space are eigenfunctions of the Laplacian in the induced metric with eigenvalue $\lambda=-\operatorname{dim}(M)$. Then the following general problem comes out in a natural way: can we classify lightlike submanifolds by means of some Laplacian differential equation involving the isometric immersion?

Our purpose in this section is to study lightlike hypersurfaces satisfying the eigenvalue equation $\Delta^{g} x=\lambda x$, where $\lambda$ is a constant and $x$ the position vector field. Our result here stands as follows.

THEOREM 6.1. Let $(M, g, S(T M))$ be a lightlike hypersurface of the Lorentzian space $\mathbb{L}^{n+2}:=\mathbb{R}_{1}^{n+2}$, endowed with a parallel screen distribution $S(T M)$ and with symmetric Ricci tensor. If $(M, g, S(T M))$ satisfies the eigenvalue equation $\Delta^{g} x=\lambda x$, then

(1) $\lambda=0$;

(2) the mean curvature function $\sigma$ vanishes identically on $M$;

(3) the shape operator $\stackrel{*}{A}_{\xi}$ of the screen distribution is trace-free with respect to the second fundamental form $B$ of $M$, that is,

$$
\sum_{\alpha=0}^{n} B\left(A_{\xi}^{*} X_{\alpha}, X_{\alpha}\right)=0
$$

Proof. If $M$ is an eigenhypersurface of $\Delta^{g}$ with eigenvalue $\lambda$, then we have, for all $x \in M$,

$$
\begin{gathered}
\lambda\langle x, N\rangle=K, \\
\lambda\langle x, \xi\rangle=K+\langle x, N\rangle \sum_{\alpha=0}^{n} B\left(\stackrel{*}{A} X_{\alpha}, X_{\alpha}\right)-\sum_{k=1}^{n}\left\langle x, X_{k}\right\rangle\left(X_{k} \cdot K\right), \\
\lambda\left\langle x, X_{k}\right\rangle=\langle x, N\rangle X_{k} \cdot K .
\end{gathered}
$$


Equation (6.2) leads to

$$
\xi \cdot K=\lambda\langle\xi, N\rangle=\lambda
$$

but we have

$$
\begin{aligned}
\xi \cdot K & =\xi \cdot\left[\sum_{\alpha=0}^{n} B\left(X_{\alpha}, X_{\alpha}\right)\right]=\sum_{k=1}^{n} \xi \cdot B\left(X_{k}, X_{k}\right) \\
& =\sum_{k=1}^{n}\left\{\left(\nabla_{\xi} B\right)\left(X_{k}, X_{k}\right)+2 B\left(\nabla_{\xi} X_{k}, X_{k}\right)\right\} \\
& =\sum_{k=1}^{n}\left\{\left(\nabla_{\xi} B\right)\left(X_{k}, X_{k}\right)\right\} .
\end{aligned}
$$

From Gauss-Codazzi equation, we have

$$
\left(\nabla_{\xi} B\right)\left(X_{k}, X_{k}\right)=\left(\nabla_{X_{k}} B\right)\left(\xi, X_{k}\right) .
$$

This leads to

$$
\left(\nabla_{\xi} B\right)\left(X_{k}, X_{k}\right)=B\left(\stackrel{*}{A_{\xi}} X_{k}, X_{k}\right),
$$

and using this and (2.15), we deduce that

$$
\xi \cdot K=\lambda=\sum_{\alpha=0}^{n} B\left(\stackrel{*}{A_{\xi}} X_{\alpha}, X_{\alpha}\right) .
$$

Then, if $\lambda=0$, the first claim in Theorem 6.1 is obvious. The second one comes from (6.2) and Definition 5.3 of $\sigma$. Finally, the third assertion follows from relation (6.9).

Now assume $\lambda \neq 0$. The differentiation of equation (6.2) with respect to $X_{k}$, $k=1, \ldots, n$, leads to

$$
X_{k} \cdot K=\lambda\left\langle X_{k}, N\right\rangle=0
$$

where we use $\bar{\nabla}_{X} N=0$ for all $X \in \Gamma(T M)$ and (2.1). Hence we get

$$
X_{k} \cdot K=0, \quad k=1, \ldots, n
$$

Substitute (6.11) in (6.4) and $\lambda \neq 0$ to get

$$
\left\langle x, X_{k}\right\rangle=0, \quad k=1, \ldots, n .
$$

Hence, there exist two smooth functions $r$ and $l$ such that the position vector field along $M$ is given by

$$
x=r(x) \xi+l(x) N
$$


Using (6.11), (6.9), and (6.2) in (6.3) leads to

$$
K+K-0=\lambda\langle x, \xi\rangle=\lambda l(x) .
$$

Hence $l(x)=2 K / \lambda$. On the other hand, we get from (6.2) that $K=\lambda r(x)$ so that $r(x)=K / \lambda$. Thus the position vector field $x$ along $M$ is given by

$$
x=\frac{K}{\lambda}(\xi+2 N)
$$

Therefore, we obtain

$$
\xi=\bar{\nabla}_{\xi} x=\frac{\xi \cdot K}{\lambda}(\xi+2 N)+\frac{K}{\lambda}\left(\bar{\nabla}_{\xi} \xi+\bar{\nabla}_{\xi} N\right) .
$$

The last term in this equality is zero $\left(\bar{\nabla}_{\xi} \xi=0, \bar{\nabla}_{\xi} N=0\right)$. Then use (6.5) to conclude that $\xi=\xi+2 N$, that is, $N \equiv 0$, which is a contradiction. Hence $\lambda=0$, and the proof is complete.

Acknowledgment. The authors would like to thank the referees for their fruitful remarks which improved the final version of the paper and Dr. Mathias Hounkpe for careful reading of the manuscript.

\section{REFERENCES}

[1] M. A. Akivis and V. V. Goldberg, The geometry of lightlike hypersurfaces of the de Sitter space, Acta Appl. Math. 53 (1998), no. 3, 297-328.

[2]___ Lightlike hypersurfaces on manifolds endowed with a conformal structure of Lorentzian signature, Acta Appl. Math. 57 (1999), no. 3, 255-285.

[3] _ - On some methods of construction of invariant normalizations of lightlike hypersurfaces, Differential Geom. Appl. 12 (2000), no. 2, 121-143.

[4] C. Atindogbe and K. L. Duggal, Conformal screen on lightlike hypersurfaces, submitted to J. Geom. Phys.

[5] C. Atindogbe, J.-P. Ezin, and J. Tossa, Lightlike Einstein's Hypersurfaces in Lorentzian Manifolds with Constant Curvature, submitted to Acta Appl. Math.

[6] _,$r$-lightlike submanifolds in the General de Sitter Space, preprint, Institut de Mathématiques et de Sciences Physiques, Porto-Novo.

[7] _ Reduction of the codimension for lightlike isotropic submanifolds, J. Geom. Phys. 42 (2002), no. 1-2, 1-11.

[8] W. B. Bonnor, Null hypersurfaces in Minkowski space-time, Tensor (N.S.) 24 (1972), 329-345.

[9] C. Burali-Forti, Fondamenti per la geometria differenziale su di una superficie col metodo vettoriale generale, Rend. Circ. Mat. Palermo 33 (1912), 1-40 (Italian).

[10] K. L. Duggal, Electromagnetic and plane waves solutions of 3-dimensional lightlike hypersurfaces, Tech. Report 95-03, Fluid Dynamics Research Institute, University of Windsor, Ontario, 1995.

[11] K. L. Duggal and A. Bejancu, Lightlike Submanifolds of Semi-Riemannian Manifolds and Applications, Mathematics and Its Applications, vol. 364, Kluwer Academic Publishers, Dordrecht, 1996. 
[12] S. W. Hawking and G. F. R. Ellis, The Large Scale Structure of Space-Time, Cambridge Monographs on Mathematical Physics, Cambridge University Press, London, 1973.

[13] D. N. Kupeli, Degenerate manifolds, Geom. Dedicata 23 (1987), no. 3, 259-290.

[14] _ _ On null submanifolds in spacetimes, Geom. Dedicata 23 (1987), no. 1, 33-51.

[15] T. Takahashi, Minimal immersions of Riemannian manifolds, J. Math. Soc. Japan 18 (1966), 380-385.

C. Atindogbe: Institut de Mathématiques et de Sciences Physiques (IMSP), Université d'Abomey-Calavi (UAC), Porto-Novo, BP 613, Benin

E-mail address: atincyr@yahoo.fr

J.-P. Ezin: Institut de Mathématiques et de Sciences Physiques (IMSP), Université d'Abomey-Calavi (UAC), Porto-Novo, BP 613, Benin

E-mail address: jp.ezin@imsp-uac.org

Joël Tossa: Institut de Mathématiques et de Sciences Physiques (IMSP), Université d'Abomey-Calavi (UAC), Porto-Novo, BP 613, Benin

E-mail address: joe1. tossa@imsp-uac.org 


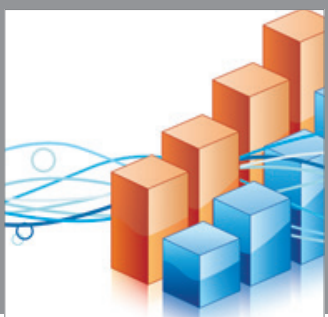

Advances in

Operations Research

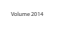

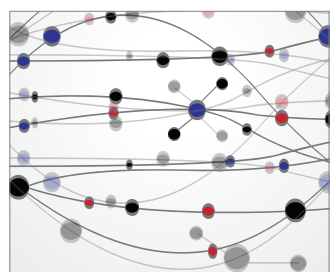

\section{The Scientific} World Journal
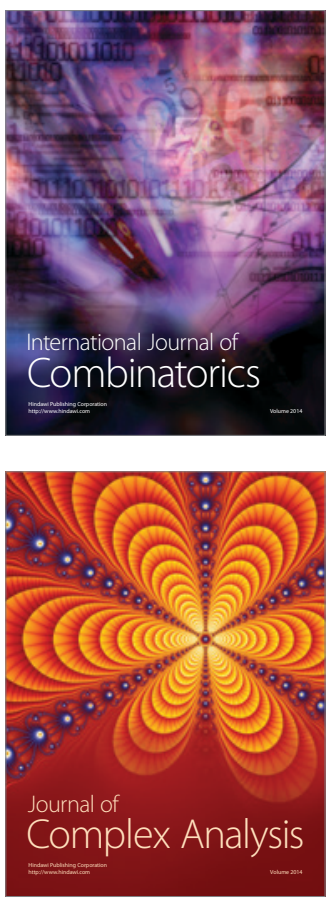

International Journal of

Mathematics and

Mathematical

Sciences
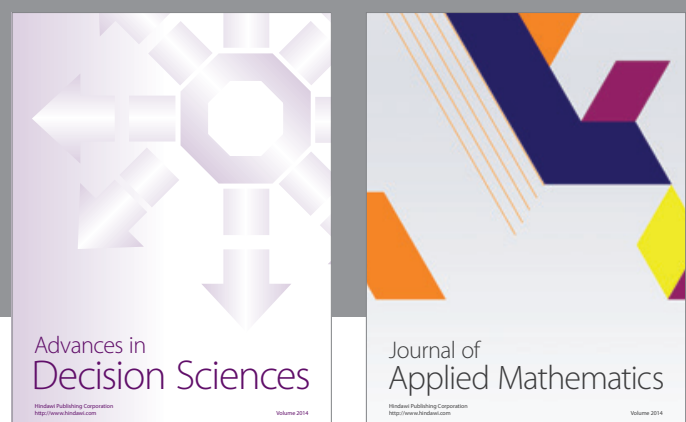

Journal of

Applied Mathematics
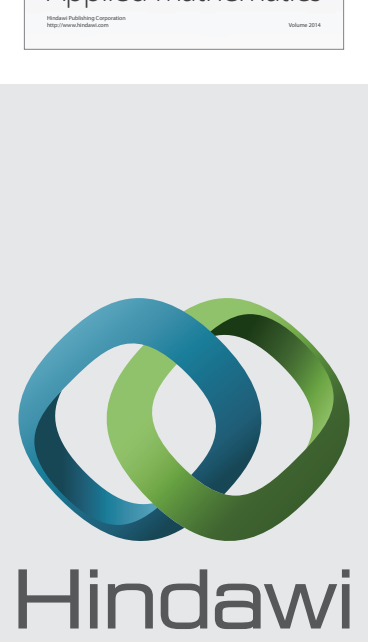

Submit your manuscripts at http://www.hindawi.com
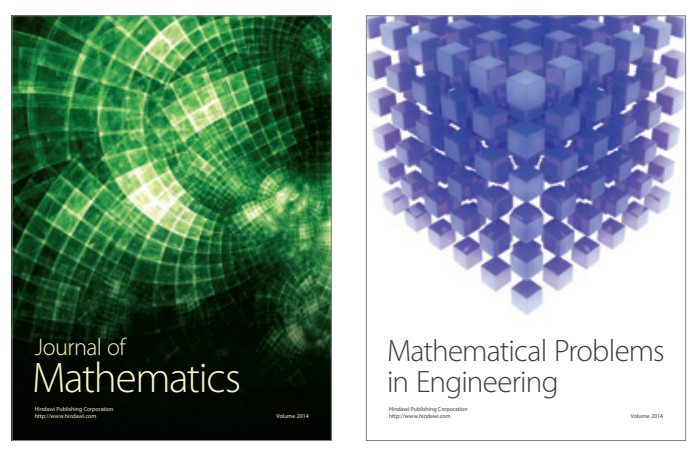

Mathematical Problems in Engineering
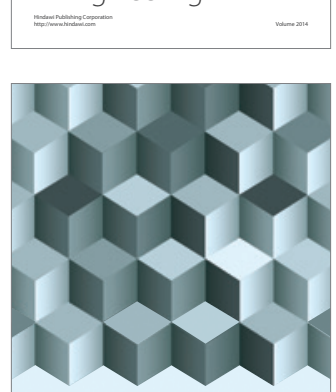

Journal of

Function Spaces
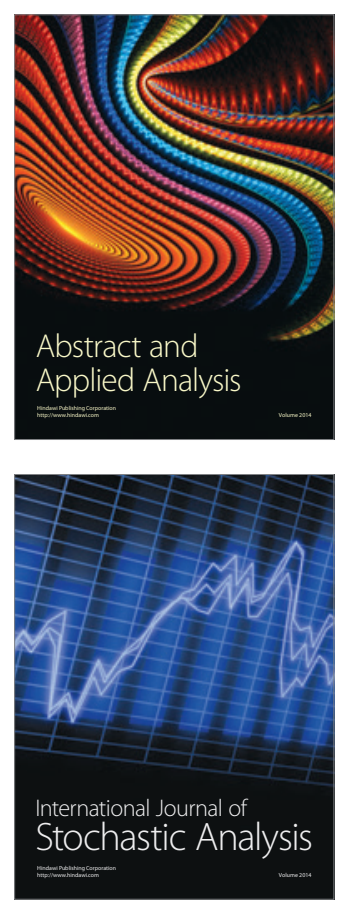

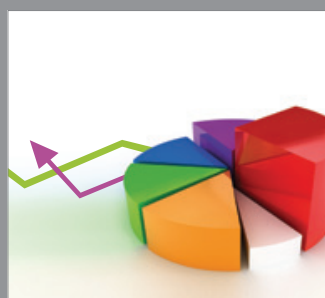

ournal of

Probability and Statistics

Promensencen
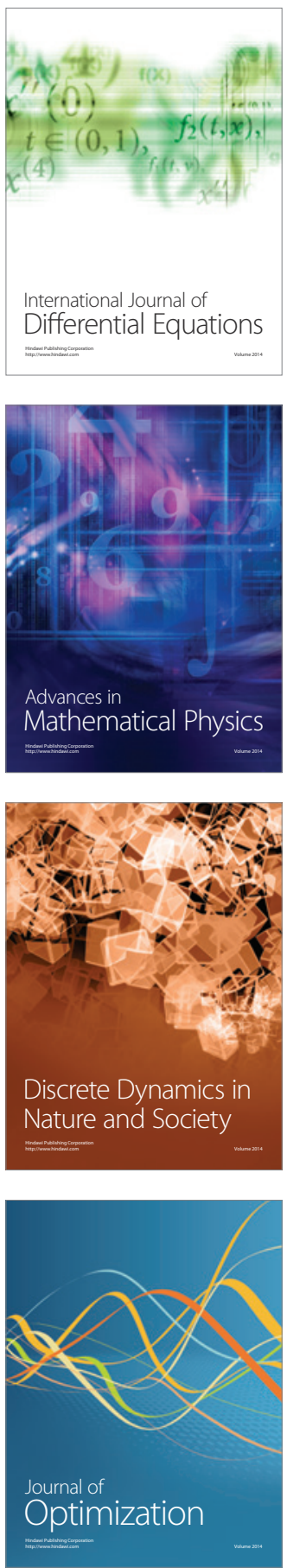